\title{
Chronic thromboembolic pulmonary hypertension after acute pulmonary embolism: to screen or not to screen?
}

\author{
Marius M. Hoeper \\ Affiliation: Dept of Respiratory Medicine, Hannover Medical School and German Center of Lung Research \\ (DZL), Hannover, Germany. \\ Correspondence: Marius M. Hoeper, Dept of Respiratory Medicine, Hannover Medical School, 30623 \\ Hannover, Germany. E-mail: hoeper.mariusamh-hannover.de
}

@ERSpublications

As the risk of CTEPH in survivors of acute pulmonary embolism is probably in the range of $0.5-1.0 \%$, screening is unlikely to be efficacious http://ow.ly/66ov30iWjnY

Cite this article as: Hoeper MM. Chronic thromboembolic pulmonary hypertension after acute pulmonary embolism: to screen or not to screen? Eur Respir J 2018; 51: 1800440 [https://doi.org/10.1183/ 13993003.00440-2018].

How many patients who survive acute pulmonary embolism will eventually develop chronic thromboembolic pulmonary hypertension (CTEPH) and is the risk sufficiently high to call for systematic screening? These two questions are obviously connected and the ongoing debate reflects a lack of robust data. Previous estimates on the incidence of CTEPH after acute pulmonary embolism have ranged from $0.1 \%$ [1] to $9.1 \%$ [2]; an almost 2-log difference. A widely regarded Italian study of 223 consecutive patients with acute pulmonary embolism found a cumulative CTEPH incidence of $3.8 \%$ after 2 years; no additional CTEPH cases were identified during long-term follow-up [3]. In contrast, a more recent series, also from Italy, that included 647 patients with acute embolism found a cumulative CTEPH incidence of $1.7 \%$ during a 3 -year follow-up $[4,5]$. A recent meta-analysis of 41 studies narrowed the numbers down but still provided conflicting results [6]: $0.56 \%$ of all patients with acute pulmonary embolism developed CTEPH over a period of 2-3 years; however, in a subset of four studies that included only survivors of acute pulmonary embolism, the CTEPH incidence was $3.2 \%$.

In this issue of the European Respiratory Journal, CoQUOz et al. [7] present the results of a prospective collaborative effort of Swiss centres which involved systematic screening for the development of CTEPH in patients diagnosed with acute pulmonary embolism between March 2009 and November 2013; follow-up ended in November 2016. Patients were excluded if they had already been diagnosed with pulmonary hypertension or suffered from other chronic illnesses causing severe dyspnoea (functional class III or IV), or if they suffered from cancer or other severe diseases with a life expectancy of less than 6 months. Overall, 1699 patients were diagnosed with acute pulmonary embolism, of whom 508 were included and followed in the CTEPH screening protocol. A three-step protocol was implemented, starting with a standardised dyspnoea assessment by telephone at 6,12 and 24 months. These telephone visits identified 97 (19.1\%) patients with persistent symptoms of functional class II or higher. In the second step, these patients underwent clinical assessment including transthoracic echocardiography, which revealed possible pulmonary hypertension in 14 patients, i.e. $2.8 \%$ of the 508 patients who underwent screening and $14.4 \%$ of the patients who had a limited exercise capacity. In the third and final step, CTEPH was diagnosed by right heart catheterisation and imaging in four of these patients, i.e. $0.79 \%$ (95\% CI $0.31-2.07 \%$ ) of the 
patients who underwent screening and $4.12 \%$ (95\% CI $1.62-10.13 \%)$ of the patients with persistent symptoms.

These numbers are considerable lower than in most previous reports, so how reliable are they? The Swiss study was certainly limited by the fact that only about $30 \%$ of the patients with acute pulmonary embolism were included in the CTEPH screening programme. Hence, some cases may have been missed, especially among patients at higher risk for developing CTEPH. Still, the authors were able to cross-reference their data with the Swiss Pulmonary Hypertension Registry and ascertained that no additional CTEPH cases were reported in their screening population.

Another factor that makes the Swiss data appear reliable is the actual number of patients diagnosed with CTEPH. In Switzerland, this number has been relatively stable at approximately 20 per year [8], and this is close to the number of CTEPH cases to be expected with an incidence of $0.79 \%$ in pulmonary embolism survivors. It was always a major drawback of previous, higher, estimates that they never matched the number of patients who were actually diagnosed with CTEPH [9]. Recent registry data from Germany found a CTEPH incidence of 5.7 per million adults [10], a figure comparable to the Swiss data as well as to observations from France [9]. Bearing in mind that only about $75 \%$ of patients with CTEPH have a medical history of previous pulmonary embolism [10, 11], these numbers are consistent with a CTEPH incidence of $0.5-1 \%$ in survivors of acute pulmonary embolism.

Do the present data justify active screening of pulmonary embolism survivors for the development of CTEPH as advocated by Coquoz et al. [7]? The answer is probably no. The Swiss approach proved feasible and could be a good model for a practical screening approach. Still, with CTEPH detected in less than $1 \%$ of the screened patients, systematic screening of all pulmonary embolism survivors would be neither medically justified nor cost-effective. Although the authors favour screening, the evidence they provide is more supportive of current guidelines, which do not recommend screening for asymptomatic survivors of pulmonary embolism but call for a thorough work-up of patients with persistent symptoms [12-14].

Notably, the Swiss data highlight, once again, that a substantial proportion of pulmonary embolism survivors remain symptomatic. About $20 \%$ of the patients had persistent exertional dyspnoea, but less than $1 \%$ had CTEPH. The clinical phenomenon of persistent limitation in cardiopulmonary function and impaired quality of life has been reported in $10-30 \%$ of the patients who survive acute pulmonary embolism and has been named "post-pulmonary embolism syndrome" [15]. CTEPH is the most dramatic but also the rarest manifestation of the post-pulmonary embolism syndrome, while the majority of these patients have a persistent reduction in their exercise capacity without signs of pulmonary hypertension [15]. The pathogenesis of this clinical syndrome is unknown and the same is true for its treatment. The ongoing FOCUS (Follow-up After Acute Pulmonary Embolism) study should provide further data on the long-term sequela of pulmonary embolism [16]. It is important to obtain more data on the clinical consequences of pulmonary embolism, not only to identify patients with CTEPH but also to learn more about the post-pulmonary embolism syndrome. Once we have a better understanding of this syndrome and know how to treat affected patients, we may reconsider systematic screening of pulmonary embolism survivors, not only for CTEPH but also for other potentially treatable conditions.

Conflict of interest: M.M. Hoeper has received fees for lectures and/or consultations from Actelion, Bayer, Gilead, GSK, MSD and Pfizer.

\section{References}

1 Fedullo PF, Auger WR, Kerr KM, et al. Chronic thromboembolic pulmonary hypertension. N Engl J Med 2001: 345: 1465-1472.

2 Marti D, Gomez V, Escobar C, et al. [Incidence of symptomatic and asymptomatic chronic thromboembolic pulmonary hypertension]. Arch Bronconeumol 2010: 46: 628-633.

3 Pengo V, Lensing AW, Prins $\mathrm{MH}$, et al. Incidence of chronic thromboembolic pulmonary hypertension after pulmonary embolism. N Engl J Med 2004: 350: 2257-2264.

4 Pesavento R, Filippi L, Palla A, et al. Impact of residual pulmonary obstruction on the long-term outcome of patients with pulmonary embolism. Eur Respir J 2017: 49: 1601980.

5 Klok FA, Hoeper MM. Predicting recurrent pulmonary embolism and chronic thromboembolic pulmonary hypertension: one more way to skin the cat. Eur Respir J 2017: 49: 1700413.

6 Ende-Verhaar YM, Cannegieter SC, Vonk Noordegraaf A, et al. Incidence of chronic thromboembolic pulmonary hypertension after acute pulmonary embolism: a contemporary view of the published literature. Eur Respir J 2017: 49: 1601792.

7 Coquoz N, Weilenmann D, Stolz D, et al. Multicentre observational screening survey for the detection of chronic thromboembolic pulmonary hypertension following pulmonary embolism. Eur Respir J 2018: 51: 1702505.

8 Mueller-Mottet S, Stricker H, Domenighetti G, et al. Long-term data from the Swiss pulmonary hypertension registry. Respiration 2015: 89: 127-140.

9 Simonneau G, Hoeper MM. Evaluation of the incidence of rare diseases: difficulties and uncertainties, the example of chronic thromboembolic pulmonary hypertension. Eur Respir J 2017: 49: 1602522. 
10 Kramm T, Wilkens H, Fuge J, et al. Incidence and characteristics of chronic thromboembolic pulmonary hypertension in Germany. Clin Res Cardiol 2018; in press [https://doi.org/10.1007/s00392-018-1215-5].

11 Pepke-Zaba J, Delcroix M, Lang I, et al. Chronic thromboembolic pulmonary hypertension (CTEPH): results from an international prospective registry. Circulation 2011: 124: 1973-1981.

12 Galie N, Humbert M, Vachiery JL, et al. 2015 ESC/ERS Guidelines for the diagnosis and treatment of pulmonary hypertension. Eur Respir J 2015: 46: 903-975.

13 Galie N, Humbert M, Vachiery JL, et al. 2015 ESC/ERS Guidelines for the diagnosis and treatment of pulmonary hypertension. Eur Heart J 2016: 37: 67-119.

14 Konstantinides SV, Torbicki A, Agnelli G, et al. 2014 ESC Guidelines on the diagnosis and management of acute pulmonary embolism: The Task Force for the Diagnosis and Management of Acute Pulmonary Embolism of the European Society of Cardiology (ESC). Eur Heart J 2014: 35: 3033-3073.

15 Klok FA, van der Hulle T, den Exter PL, et al. The post-PE syndrome: a new concept for chronic complications of pulmonary embolism. Blood Rev 2014: 28: 221-226.

16 Konstantinides SV, Barco S, Rosenkranz S, et al. Late outcomes after acute pulmonary embolism: rationale and design of FOCUS, a prospective observational multicenter cohort study. J Thromb Thrombolysis 2016: 42: 600-609. 